\title{
Model-based digital pianos: from physics to sound synthesis
}

\author{
Balázs Bank, Member, IEEE and Juliette Chabassier
}

October 12, 2018

\begin{abstract}
Piano is arguably one of the most important instruments in Western music due to its complexity and versatility. The size, weight, and price of grand pianos, and the relatively simple control surface (keyboard) have lead to the development of digital counterparts aiming to mimic the sound of the acoustic piano as closely as possible. While most commercial digital pianos are based on sample playback, it is also possible to reproduce the sound of the piano by modeling the physics of the instrument. The process of physical modeling starts with first understanding the physical principles, then creating accurate numerical models, and finally finding numerically optimized signal processing models that allow sound synthesis in real time by neglecting inaudible phenomena, and adding some perceptually important features by signal processing tricks. Accurate numerical models can be used by physicists and engineers to understand the functioning of the instrument, or to help piano makers in instrument development. On the other hand, efficient real-time models are aimed at composers and musicians performing at home or at stage. This paper will overview physics-based piano synthesis starting from the computationally heavy, physically accurate approaches and then discusses the ones that are aimed at best possible sound quality in real-time synthesis.
\end{abstract}

\section{Introduction}

The piano is arguably one of the most important and most versatile musical instruments in the Western world. In classical music, it can stand for its own (solo pieces), can work as a lead instrument (piano concertos), or accompany other soloists. In addition, it has a special role in jazz and other popular genres.

However, due to its size, weight and price, not everyone can afford to own such an instrument. Some of these factors are less critical for upright pianos, but the volume of

${ }^{*}$ B. Bank is with the Department of Measurement and Information Systems, Budapest University of Technology and Economics, H-1521 Budapest, Hungary (e-mail: bank@mit.bme.hu).

${ }^{\dagger} \mathrm{J}$. Chabassier is with the Magique 3D Inria team, Inria Bordeaux Sud Ouest and Applied Mathematics Laboratory of the University of Pau, France (e-mail: juliette.chabassier@inria.fr).

${ }^{\ddagger}$ Manuscript received October 12, 2018, revised October 12, 2018. 
the instrument can be still a problem when practicing at home or transporting it as a performing musician. Therefore, digital piano synthesizers have been developed. They are usually termed "digital pianos", which discriminate them from early electromechanical instruments, such as Fender Rhodes or Wurlitzer, called "electric pianos".

Most digital pianos available at the market are using sampling technology, meaning that the sounds of an acoustic piano are recorded and then played back when a key is pressed. In the early times limited memory sizes meant compromised quality, but nowadays it is possible to sample each note individually with various key velocities / loudness levels.

However, some phenomena, like the free vibration of the strings when the sustain pedal is pressed, the coupling between the strings of the sounding notes, or the restrike of an already sounding string, cannot be easily and concisely reproduced by recorded samples. Another desired factor that cannot be easily fulfilled in sampling-based pianos is the possibility for the player to continuously alter the properties of the piano sound, e.g., by changing the hardness of the hammer, the tuning of the string, or the position of the (virtual) recording microphones. In sampling technology, these changes can only be faithfully reproduced by having different sample sets for all the different scenarios.

With ever increasing computational capacity of computers and digital signal processors, a different approach for synthesizing piano sound has become possible: physical modeling synthesis. Physical modeling aims at reproducing the functioning of the whole instrument instead of resynthesizing some recorded sound samples (which does not exploit any a priori knowledge on their physical origin). Therefore in theory it should allow a more faithful virtual reproduction of the piano with a better responsiveness to the actions of the piano player.

Besides creating a more portable and affordable substitute for the acoustic piano, modeling the piano can be useful for various other reasons. First, modeling is interesting for the physicists and engineers who want to capture the physics of the instrument, the phenomena happening at each step of the sound production, the reasons for historical evolutions. It can therefore help the community rationalize and understand the empirical statements heard for centuries. Then, for the manufacturers who want to make the piano evolve, on the acoustical and structural point of view, based on practical (making processes, availability, cost and workability of materials, technical evolutions) and musical (aesthetic quality of the sound, playability, dynamic response) motivations. Finally, for the composers and players who use virtual synthesized pianos that respond in real time to their playing on the keyboard, and are motivated by the tunability and playability of the virtual instrument and the realism of the sound. Therefore, the requirements made on the developed digital pianos differ from one community to the other, especially regarding the part of the physics that "cannot be heard" but is coming from structural requirements regarding the piano construction. Conversely, manufacturers sometimes have to choose processes for practical reasons, which have an impact on the sound that could be implemented differently. The choice of modeling the physical phenomena faithfully or only reproducing their effect on the sound depends on the final reached goal: realism of the produced sound, or physical 


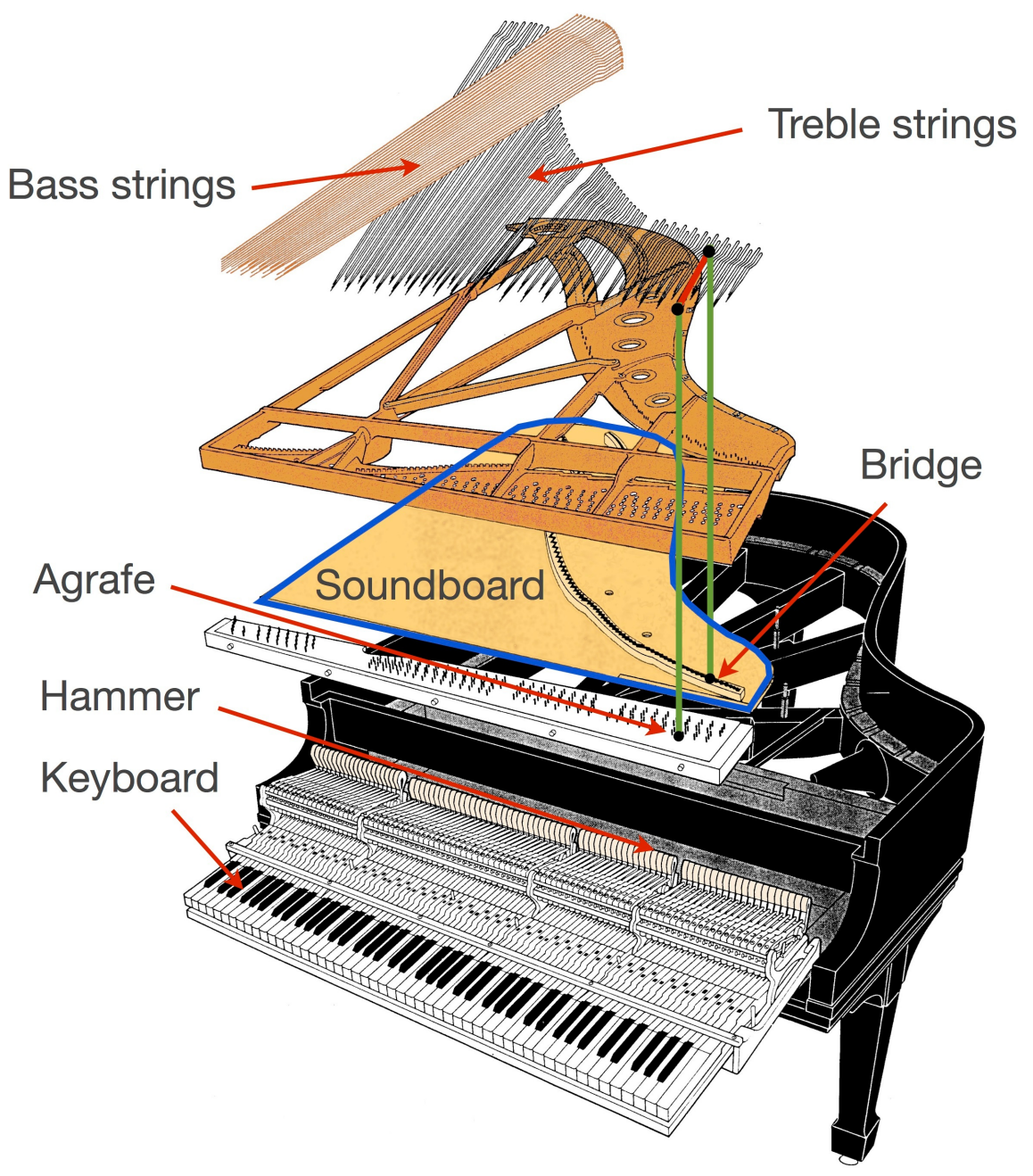

Figure 1: Exploded view of a grand piano. 
accuracy at each point of the instrument.

The aim of our paper is to approach physics-based piano synthesis along these different possible goals: from heavy, accurate, physical modeling techniques to real-time sound synthesis techniques aimed at the best possible player involvement and experience. Note that the methodology of piano measurements is out of the scope of this paper, for that, see [1,2] for example.

\section{Physics of the piano}

When a pianist strikes a key of a grand piano (see Fig. 1), a complex mechanism transmits this motion to the hammer, a small piece of wood covered with felt, which is attached at the end of a thin wood shank. After a controlled phase during which the hammer motion is directly related to the key motion, the hammer travels freely. This is until the hammer strikes one, two or three strings, depending on the played note. The strings are made of steel and the bass ones are wounded with copper. They go from the tuning pin, are blocked at the agrafe, pass over the bridge through two pins and join another blocking point linked to the steel frame. The vibrating part of the string, which is struck by the hammer, is between the agrafe and the bridge. However, the other parts of the string can vibrate by sympathy and originate the "duplex stringing" effect. Interesting properties of the piano strings $[2[6]$ are the fact that they exhibit: inharmonicity, beating (see for instance partial 5 in Fig. 2) and two-stage decay for various reasons (double string polarization, coupling with the soundboard, coupling at the bridge) and a nonlinear behavior that couples transversal waves (orthogonal to the string's elongation at its rest position) to longitudinal waves (parallel to the string's elongation at its rest position), and which conducts to precursors in the sound and to phantom partials in its spectral content (see the linear and nonlinear partials in Fig. 2). All effects contribute to the sound and the physics of the piano, and should be represented in a faithful model. When the string vibrations reach the bridge, which is composed of one or many long pieces of wood, they are transmitted to the soundboard via a complex coupling mechanism.

Experiments [5] suggest that both transversal and longitudinal string oscillations are transmitted to the soundboard. The soundboard is a pre-strained shell of glued laminated spruce wood, carrying long wooden ribs on its underside, and one or several bridges on its upper side. The strings, passing over the bridge, exert a load that constrains the soundboard and makes it flat. Finally, the soundboard radiates in the surrounding air via acousto-mechanical coupling. The soundboard is attached to the wooden case called the rim, where the keyboard rests as well, while the strings are attached to the steel frame. The case can be closed or open by a thin lid. Experiments also show [7] that all these parts vibrate as well and contribute more or less to the piano sound, depending on the range of the played notes. Finally, other features as dampers and una corda, can be operated by the keys or pedals, which are very important for playability and musical expression. 


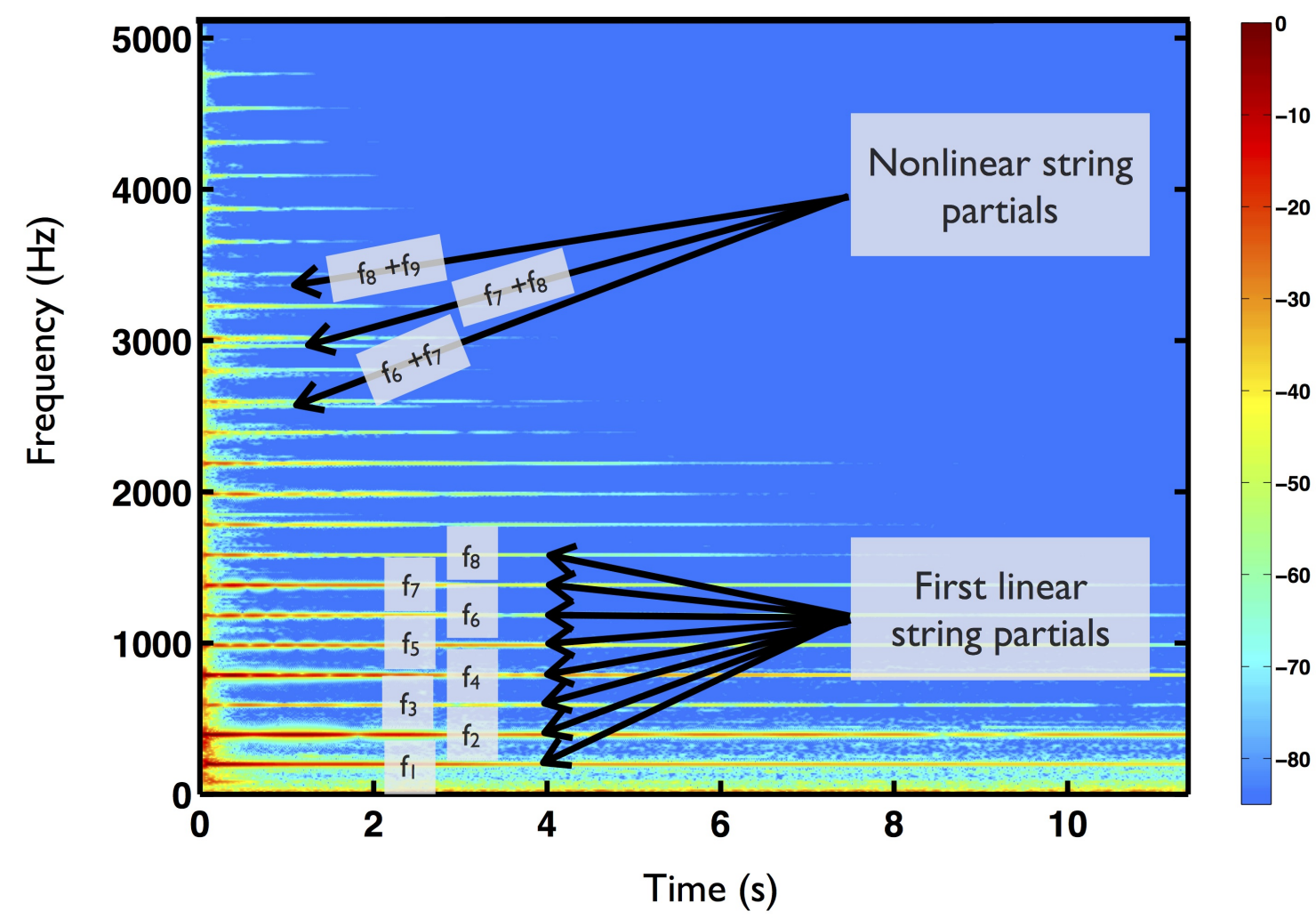

Figure 2: Spectrogram of a recorded $G_{3}$ fortissimo tone of a Steinway D grand piano. Linear partials are not exact harmonics because of dispersion in the string. Beating appears (see the amplitude modulation of partial 5 , for example) because three strings vibrate together for this note. Nonlinear "phantom" partials are visible between the quasi-harmonic series. Damping is frequency dependent: upper partials decay faster than lower ones. Finally, the soundboard contributes with a background shock sound. 


\section{Comprehensive physical modeling of the piano}

Although it would be theoretically possible to write the 3D equations satisfied by each and every part of a grand piano, and try to simulate the displacements, stresses and pressures at every point of the piano and the surrounding air, the resulting model would be simply impossible to solve on existing computational facilities and / or in a reasonable time. Therefore, and anticipating on the need to design reduced models for real-time sound synthesis, each part of the piano should be modeled with the most adequate and concise description. Several comprehensive models can be found in the literature, from the most simple ones having only a hammer and a string $8-10$ to more elaborate ones including a soundboard and sound radiation [11 and to more extensive ones taking the nonlinearity of the string, and all the coupling phenomena into account [12]. These methods solve the equations in the space and time domain, such that the output of the computation is the displacements and stresses at each point of the parts of the piano, at each time. Special care must be given to the design of stable and accurate numerical methods, which is not a simple task in the presence of nonlinear behavior and for coupled systems.

\subsection{Strings}

The ideally flexible, lossless, and unterminated (infinite) string can be described by the d'Alembert equation (1a-1), where $y=y(x, t)$ is the transversal displacement of the string at position $x$ and time $t, \mu$ is the linear mass density (mass per unit length) and $T_{0}$ is the tension of the stretched string [13]. For treble strings made of steel the linear mass density is the product of the steel density and the cross section area $S$. For bass strings, which are wounded with copper, the string mass must be measured from which an effective linear density is computed by dividing with the string length.

Equation (1a-1) has the form of a "1D wave equation" that describes various wave phenomena: the longitudinal vibration of linear and homogeneous solids, the vibration of air in tubes, and ideal wave propagation in lossless electrical transmission lines (telegrapher's equation).

The vibrating part of the string is terminated by the agrafe and the bridge in the case of the piano. In a first approximation, we can assume that these terminations $x=0$ and $x=L$ are rigid, which leads to null displacement boundary conditions (1b).

In addition, the string vibration decays due to internal and radiation losses of the string, in a frequency-dependent way, which can be taken into account by adding a constant term and a frequency-dependent term (1a-2), see [10].

Piano strings are quite thick in comparison to other string instruments (guitar, violin, etc.), thus, they cannot be assumed as perfectly flexible and start to borrow some of the vibrational behavior of metal bars. This introduces a stiffness which makes high frequency waves travel faster than low frequency waves, which is called dispersion. This physical behavior makes the overtones of the piano deviate from the perfect harmonic series com- 
mon to most musical instruments. This "inharmonicity" is a very important perceptual characteristic of the piano tone [14]. Several models can account for stiffness, which differ by their frequency range validity. The most common model in the case of the piano is the Euler-Bernoulli model that adds the term (1a-3) [9, 13], where $E$ is the Young's modulus of the string. This model considers that the sections of the string are rigid and stay orthogonal to the string's neutral axis. This model must be completed by additional boundary conditions, which in a first approximation correspond to rigid terminations (1c). Timoshenko's model, used in [12, also considers rigid sections but allows them to rotate around their rest position, and results in a system of two unknowns instead of an augmented scalar wave equation.

A last term (1a-4) describes all the external forces acting on the string, like a hammer striking the string.

A possible comprehensive resulting string model accounting for all cited phenomena can be written as

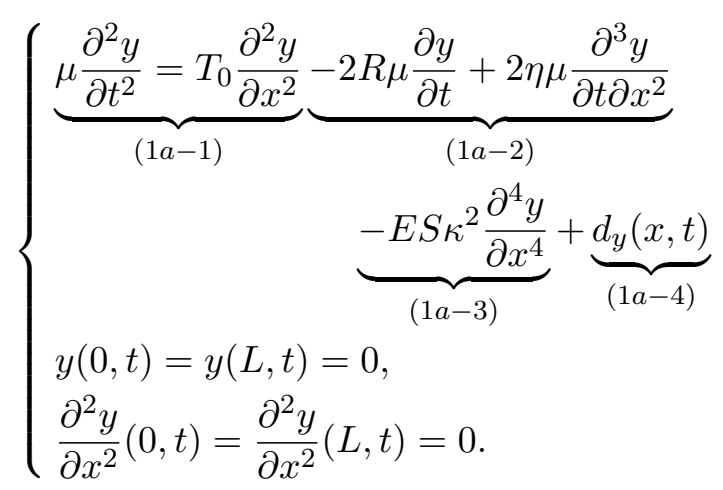

This system can then be discretized using FD in space and time, as in [9], or FEM-FD as in [12]. Let us illustrate the resulting algorithm when we solve equations ([1a-1)-1b) with a Finite Elements (FEM) discretization for the space coordinate and a Finite Difference (FD) discretization for the time coordinate. The basic idea of FEM-FD method is to seek the unknown $y(x, t)$ at regular times $t_{n}=n \Delta t$, where $\Delta t$ is called the time step of the method, and $n$ an integer. The space discretization relies on a mesh which can be regular or not, depending on the physical problem. The mean space step is called $h$. On each element of the mesh, the solution is sought as a linear combination of high order polynomial functions which are called basis functions (see an illustration at order 3 in Fig. 3, at order one they are triangle-shaped "hat functions"), therefore the unknown becomes the vector $u_{k}[n]$ for $K$ nodal amplitudes at each time instant $n$. The method also relies on the choice of quadrature formulae in order to compute integrals terms [15] and it happens that the adequate choice of Gauss-Lobatto quadrature along with basis functions which are chosen as Lagrange interpolation polynomials on these points leads to an explicit update 


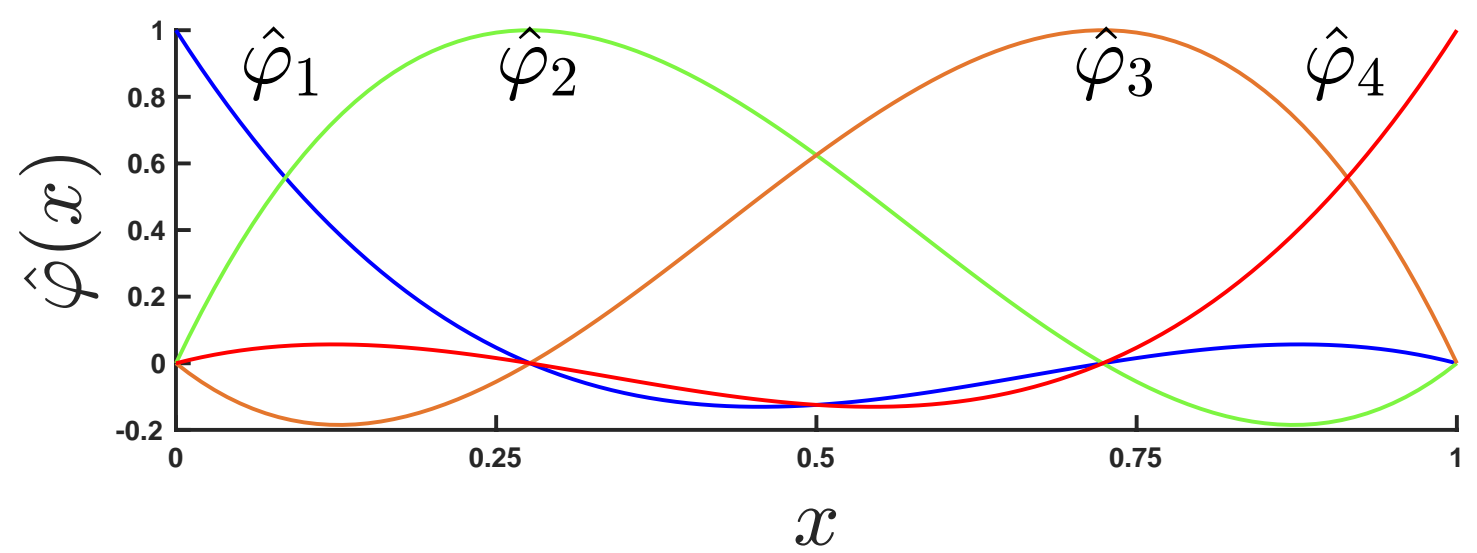

Figure 3: Finite Elements basis functions of order 3 on the unit interval: Lagrange interpolation polynomials based on Gauss-Lobatto points.

algorithm:

$$
u_{k}[n+1]=2 u_{k}[n]-u_{k}[n-1]+\frac{\Delta t^{2}}{m_{k}} \sum_{j=1}^{K} A_{k, j} u_{j}[n]
$$

where $A_{k, j}$ is the so called "stiffness matrix", a sparse matrix whose band size is related to the order of the FEM, and $m_{k}$ is the $k^{t h}$ mass coefficient. Their value depend on $h$ and on the physical coefficients of the equation $T_{0}$ and $\mu$. Finite Differences in space can actually be interpreted as first order FEM. Increasing the order of Finite Elements decreases exponentially the numerical error induced by the spatial discretization on the solution.

Additionally to the transversal wave, the presence of longitudinal waves in the string has important effects both in the time and frequency domains (referred to as nonlinear precursor and phantom partials). A physical model accounting for a geometrically exact tension is derived in 13 and leads to a nonlinear coupling between transversal and longitudinal waves involving an square root. Although this model has very attractive mathematical properties, Taylor expansions have been performed 16, 17] in order to understand the effects at first and second orders and to ease computational difficulties. It turns out that the longitudinal wave $v=v(x, t)$ is the solution of a d'Alembert equation forced by a nonlinear term that depends on $y$ :

$$
\mu \frac{\partial^{2} v}{\partial t^{2}}=E A \frac{\partial^{2} v}{\partial x^{2}}+\frac{E A-T_{0}}{2} \frac{\partial}{\partial x}\left(\frac{\partial y}{\partial x}\right)^{2}
$$

Reciprocally, the transversal wave equation is forced by a higher order nonlinear expression of $v$ and $y$.

Such expansions are not performed in 12 where a Finite Elements space discretization is proposed along with an energy-consistent time discretization for a stiff and geometrically 
exact nonlinar string. Finally, other models allow to eliminate the longitudinal wave by considering a nonlinear and non-local string equation [18].

The main displacement of the string is in the direction of the hammer strike. However, because of slight imperfections of the string, and complex boundary conditions, the string also vibrates in the orthogonal polarization [19]. This double polarization is one possible explanation of the observed amplitude modulation (two-stage decay and beating, see for instance the partial $f_{5}$ in Fig. 22 of piano sounds. Another explanation that when a single key is struck, two or three strings are sounded which are slightly detuned [3] (except for the lowest octaves).

One last feature linked to strings is the presence of dampers, long felt strips that always contact the strings except when the sustain pedal is operated, or when the corresponding key is pressed. The main effect of the dampers is a dissipative effect, but a realistic model should account for the dynamic interaction between dampers and strings, and the fact that the dissipation is not perfect. The highest notes of the piano are not equipped with dampers, therefore the corresponding strings are always vibrating.

Finally, the non-excited parts of the strings are mainly damped with felt but some piano makers choose not to damp them, in order to create a resonance which contributes to the overall piano sound. This is called "duplex stringing".

\subsection{Action and hammer}

The action that converts the key motion into the hammer motion is very complex 20,21 and relies on many lever arms made of wood, joined with rollers covered with felt. At the end, the hammer head is a piece of wood covered with felt that crushes when interacting with the string.

In a first but efficient approximation, the three-dimensional deformation of the piano hammer head can be described as a small mass connected to a nonlinear 0D spring that contacts the string around a point $x_{\mathrm{h}}$. The equations describing the hammer-string interaction are as follows 22, 23]:

$$
\begin{aligned}
& F_{\mathrm{h}}(t)=F(\Delta y)=\left\{\begin{array}{ll}
K_{\mathrm{h}}(\Delta y)^{P_{\mathrm{h}}} & \text { if } \Delta y>0 \\
0 & \text { if } \Delta y \leq 0
\end{array},\right. \\
& F_{\mathrm{h}}(t)=-m_{\mathrm{h}} \frac{d^{2} y_{\mathrm{h}}(t)}{d t^{2}},
\end{aligned}
$$

where $F_{\mathrm{h}}(t)$ is the interaction force, $\Delta y=y_{\mathrm{h}}(t)-y_{\mathrm{s}}(t)$ is the compression of the hammer felt, $y_{\mathrm{h}}(t)$ is the position of the hammer, and $y_{\mathrm{s}}(t)$ is the position of the string at the excitation point $x_{\mathrm{h}}$ (i.e., $y_{\mathrm{s}}(t)=y\left(x_{\mathrm{h}}, t\right)$ ). The hammer mass is denoted $m_{\mathrm{h}}, K_{\mathrm{h}}$ is the hammer stiffness coefficient, and $P_{\mathrm{h}}$ is the stiffness exponent. A hysteretic behaviour can also be modeled by adjusting the force $F_{h}(t)$ with a dissipative term, accounting for the discrepancy between the hammer compression and relaxation phases. 
The bending of the hammer shank, a small wooden 1D beam that holds the hammer head, has been investigated in order to understand one possible mechanism through which the pianist's touch influences the piano sound [24].

\subsection{Bridge and soundboard}

Production of soundboards takes years, during which the wood is dried and boards are glued together along the wood (spruce) fibers. The resulting plate is given a curvature called the "crown", that is supposed to compensate for string load, when they are put in tension. As a result, the soundboard looks flat, but is pre-strained by the strings. This feature, called the crown, has been studied in 25 but is usually neglected in soundboard models, which describe the soundboard with usual plate equations as Kirchhoff-Love or ReissnerMindlin [26] systems. Both models exhibit good mathematical properties, although the last is more suitable for usual Finite Elements space discretization. These models can easily account for the fact that the soundboard is thicker at its center than its edges. Since the wood is orthotropic (the waves travel at different velocities in orthogonal directions, due to the wood fibers), makers arrange ribs under the soundboard in order to restore, at least at low frequencies, a certain isotropy.

An accurate model of the ribs would be to consider each one as a beam coupled to the plate, but at low frequencies it is sufficient to model their presence as a local change of thickness of the soundboard plate. The soundboard is attached to the rim in a non trivial manner, making the boundary conditions difficult to express, ranging between simply supported and clamped. Finally, waves are damped by various phenomena inside the soundboard. Dissipation phenomena are way more complex than wave propagation, and therefore creating comprehensive dissipation models would require first, a tedious parameter fitting work, and second, a disproportionate computational effort with respect to the rest of the piano. This is why the dissipation is often described (and measured, see 27$]$ ) on the basis of the vibration modes of the soundboard. An efficient computational process, proposed in [12], consists in pre-computing the modes using a Finite Elements method, and to use these modes (see Fig. 4) as a representation basis for the soundboard displacement, by adding a modal dissipation suggested by experiments.

The bridge transmits the string vibrations to the soundboard, and vice versa. The bridge itself consists in a laminated maple or beech beam, which can be modeled with one-dimensional beam equations (Euler-Bernoulli or Timoshenko, for instance). However, a model with accurate string-bridge-soundboard coupling is still lacking, and most existing models consider the bridge as an ideal coupling feature between the strings and the soundboard. Recent attempts to develop more complex models are described in [28]. It is possible that the vertical vibration of the string is transmitted via solid coupling, while the longitudinal vibration exerts a torque, which induces a shear wave in the soundboard. Moreover, the pins through which the strings pass could also be responsible for the transmission of the orthogonal polarization. Finally, the bridge vibration shall couple remote 

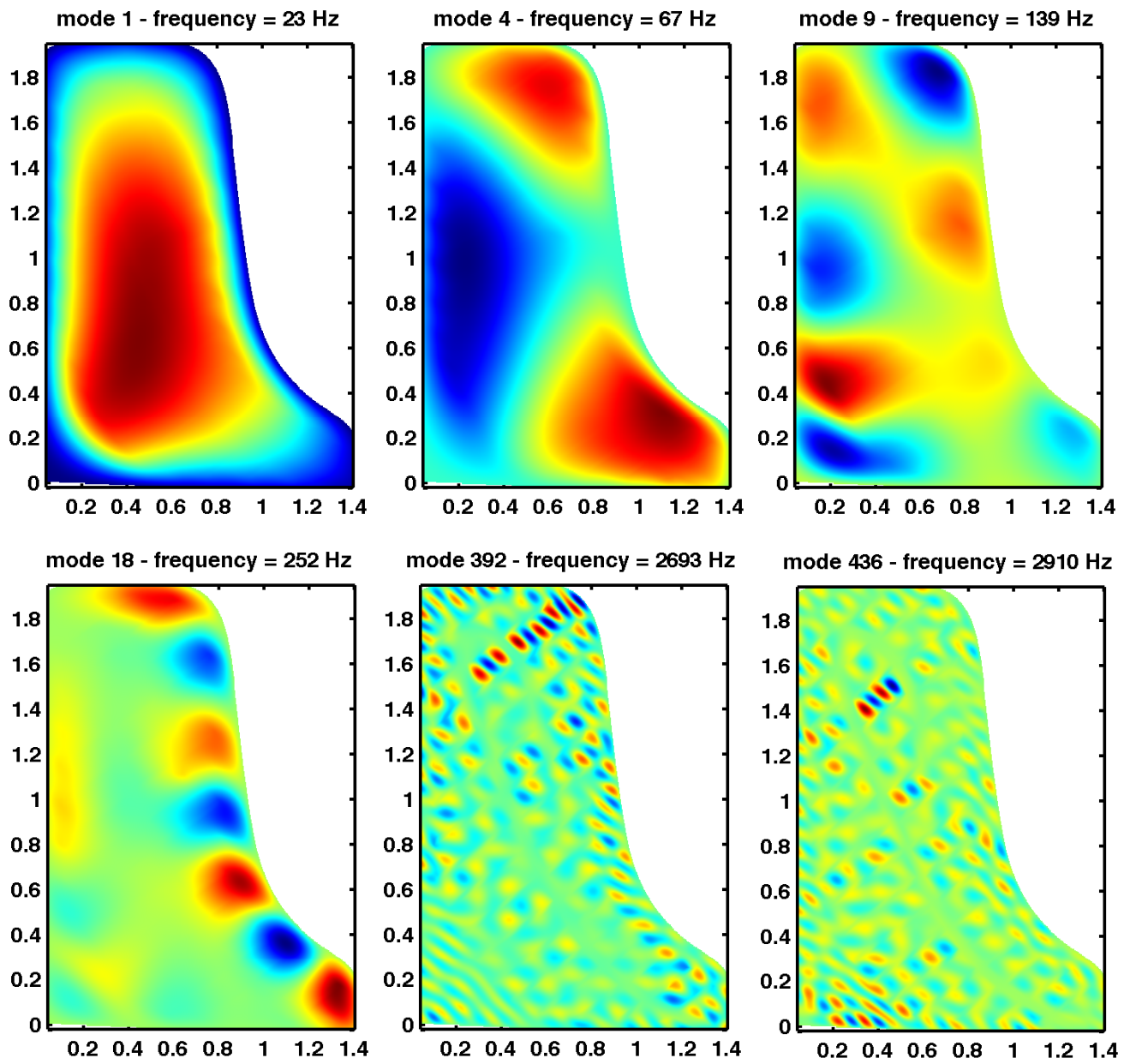

Figure 4: Some computed soundboard modes from [12. Low frequency modes are not sensitive to the fine geometrical features, while high frequency modes are trapped between the ribs. 
strings when played at the same time, and emphasize sympathetic vibrations.

\subsection{Sound radiation in the air}

Sound radiation in the air can be faithfully modeled by the three-dimensional linear acoustic wave equation

$$
\partial_{t}^{2} p-c^{2} \Delta p=0
$$

where $p=p(\mathbf{x}, t)$ is the sound pressure at a point $\mathbf{x}$ of the open space $\mathbb{R}^{3}$ and time $t, c$ is the sound celerity, and $\Delta$ is the Laplace operator. The presence of the piano rim and lid can, to a first approximation, be considered as obstacles to sound propagation, although a refined model could account for their respective vibrations. The soundboard constitutes a singular surface in the propagation free space, where the mechanical normal velocity of the soundboard is set equal to the acoustical normal velocity. Reciprocally, the pressure jump between the upper and lower part of the soundboard exerts a load on the soundboard, which is modeled as a force at the rhs of the soundboard's equation.

These equations can easily be discretized in space using Finite Differences [11], but this method does not capture well the geometrical details of the rim and soundboard, and leads to severe spurious numerical dispersion. A more accurate possibility is to use high order spectral Finite Elements 12 . The room must be artificially truncated in order to limit the computational domain, which can be done using Absorbing Boundary Condition or Perfectly Matched Layers. The acoustic pressure and velocity at a distant point can be recovered by analytical formulae based on closed surfaces (retarded potentials). Another option would be to pre-compute the impulse responses at several points around the soundboard, but this neglects the reciprocal coupling between the sound propagation and the soundboard.

\subsection{About time discretization and computational efficiency}

The resulting mechanical system of this modeling process is a nonlinear coupled system involving many dimensions $(0 \mathrm{D}, 1 \mathrm{D}, 2 \mathrm{D}, 3 \mathrm{D})$ with reciprocal interactions. The time discretization must be performed by ensuring numerical stability, which is not straightforward in this complex context, but also seeking the best possible computational efficiency. One possibility is to rely on energy-based techniques, as in [29] for the string, or [11,12] for the whole piano. The final algorithm can be run in parallel on computational facilities and it currently requires 24 hours of computation on 300 processors to get accurate displacements, strains, pressures everywhere in and around the piano during 1 second of physical time 12 .

\subsection{Overview and drawbacks}

To conclude, these comprehensive physical models give access to all internal states of the instrument and thus can be used to better understand the physics of the piano. In addition 
to estimating the effects of changes in the geometric or material properties of the virtual instrument 30,31], it is also possible to model a piano that does not exist, or does not exist anymore in playing condition. Many features are still missing from existing comprehensive simulation tools, like key restrike, sympathetic strings, duplex stringing, aliquots, dampers, una corda pedaling, lid positioning, etc. Some of these are relatively simple additions, while others would lead to a significant increase in computation time.

On the other hand, listening to the obtained sounds is disappointing not only because of the aforementioned missing features, but also because the ear is very sensitive to decay rates [32], which are linked to dissipation phenomena that we do not yet understand well. In a sense, listening to these sounds gives us an auditory measure of what we understand today about the physics of the piano.

\section{Reduced models for sound synthesis}

Sound synthesis, on the contrary, requires the best possible perceptual quality at relatively low computational cost. Therefore the idea is to slightly depart from "physicality" in favor of sonic realism. This is done by applying models that can be easily fine-tuned based on the analysis of recorded piano tones, and also manually tuned by experts. While the fine structure of the model parameters are set during model creation, the user still has control over the general properties of the piano sound (overall string decay, inharmonicity and detuning, hammer mass and hardness, etc.) in a physically meaningful way. Note that in comprehensive piano models aimed at understanding the physics of the instrument parameterizing the model based on recorded piano sounds would be unacceptable, since it would prevent from understanding how the physical parameters of the instrument (string mass and stiffness, soundboard geometry and material, etc.) influence the piano behavior.

\subsection{Efficient string modeling by digital waveguides}

Since an acoustic piano has more than 200 strings, it is crucial to model them effectively. One of the most efficient way of string modeling is digital waveguides [33]. The timedomain solution of the lossless wave equation (1a-1)-1b in an infinite medium was given by d'Alembert in 1747 :

$$
y(x, t)=f^{+}(c t-x)+f^{-}(c t+x),
$$

meaning that the vibrational behavior of the ideal, unterminated string can be described by two independent waveshapes traveling in the opposite directions. This is the so called "traveling-wave solution". The idea of digital waveguides is that instead of numerically solving the wave equation (1a) as with the finite element method in (2), it implements its analytical solution (6) directly.

The efficiency of the method comes from the fact that the sampled versions of the two waveshapes can be easily stored in two arrays in computer memory whose content are 
shifted to the right or to the left at each time sample. In signal processing terms, the two traveling waves are represented by two delay lines. The string displacement $y\left(x_{m}, t_{n}\right)$ at discrete position $x_{m}$ and discrete time $t_{n}$ is the sum of the two delay lines. This is displayed in Fig. 5 (a).

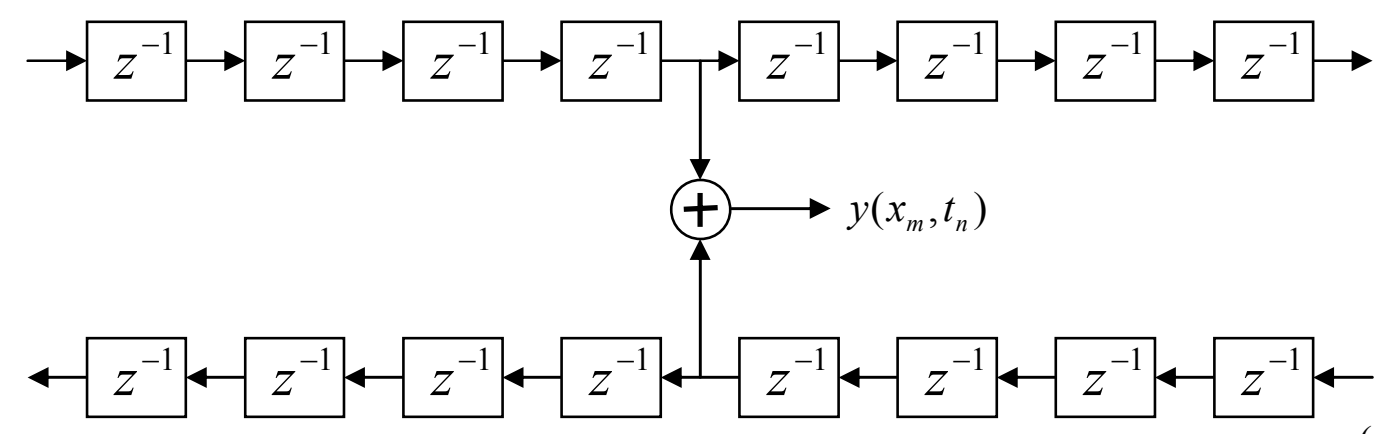

(a)

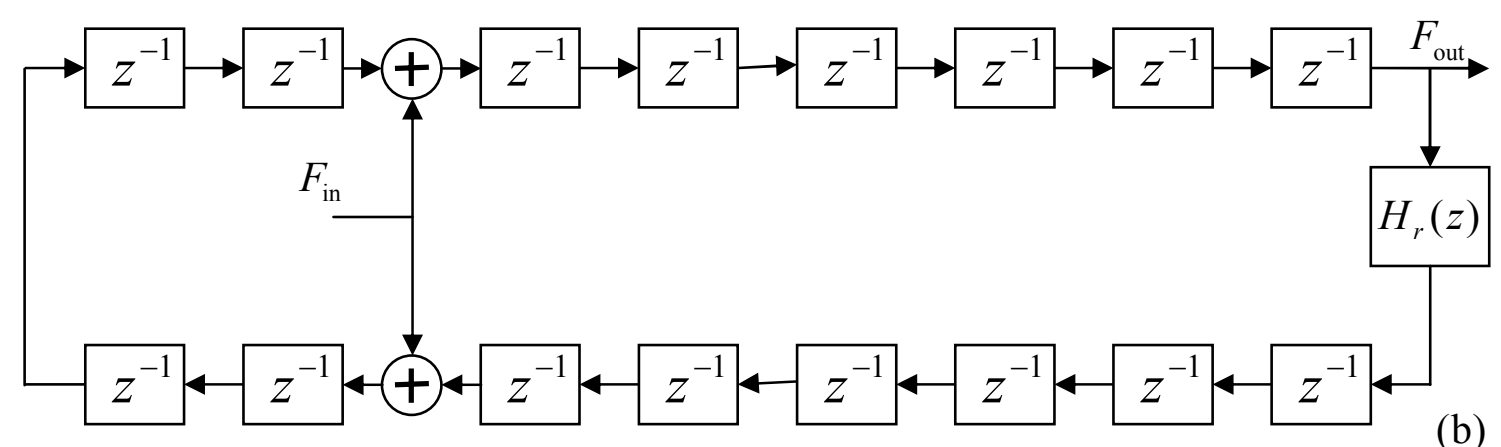

Figure 5: Digital waveguide modeling: (a) model of an infinite ideal string and (b) model of a terminated string with reflection filter, force input and output.

The structure of Fig. 5 (a) describes the case of the infinite string, but terminating the string with perfectly rigid boundaries creates wave reflections having opposite sign [13]. This can be easily modeled in digital waveguides by simply feeding back the output of one delay line to the other with opposite sign.

Real strings exhibit losses and dispersion, as discussed in Sec. 3.1 . Losses can be easily incorporated in the model by inserting attenuation filters between the delay elements in Fig. 5 (a).

Modeling the dispersion is somewhat more complicated since it actually requires that the waves travel at a frequency-dependent speed. Since the points between the delay elements correspond to the sampled physical positions along the string, this can be accomplished only if the delay elements can shift the signal by a fractional sample, not only by 
one, as for unit delays. This can be implemented by replacing the unit delays with allpass filters whose delay depends on frequency [34].

However, inserting individual loss and dispersion filters between the unit delays would complicate the structure and we would loose all the computational benefits coming from the fact that the d'Alembert solution of the wave equation is discretized.

Before proceeding with this issue, the input and the output of the string model should be added: the string is excited by the hammer strike acting at a single position of the string, this is displayed by $F_{\text {in }}$ in Fig. 5 (b). The string vibration is transferred to the soundboard through the bridge, thus, the force needs to be computed at one of the endpoints of the string. This is displayed by $F_{\text {out }}$ in Fig. 5 (b) with a digital waveguide that now transmits force waves.

Accordingly, rather than computing the string shape at all positions, we are only interested in the behavior of the string between its input and output. Therefore, we can lump the effects of losses and dispersion occurred at one round trip of the waves in the string into a single filter. This is called "reflection filter" and displayed as $H_{\mathrm{r}}(z)$ in Fig. 5 (b). Consolidating all the losses and dispersion to a single point greatly increases the efficiency, since delay lines can be implemented at almost zero cost by using circular buffers, and a single relatively low-order filter is also efficiently realized in DSPs. The transfer function of the digital waveguide is

$$
H_{\mathrm{wg}}(z)=\frac{F_{\mathrm{out}}(z)}{F_{\mathrm{in}}(z)}=H_{c}(z) \frac{1}{1-z^{-N} H_{\mathrm{r}}(z)},
$$

where $H_{\mathrm{c}}(z)$ is a comb filter coming from the fact that the force input is acting at two points on the delay line with opposite sign, and $N$ is the total length of the delay line.

The modal frequencies of the digital waveguide can be estimated by finding the local maxima of the transfer function $H_{\mathrm{wg}}(z)$ where the feedback structure has very high (almost infinite) gain. These are the frequencies where the denominator is close to zero, that is, $z^{-N} H_{\mathrm{r}}(z) \approx 1$. The magnitude of the reflection filter $\left|H_{\mathrm{r}}(z)\right|$ is close to unity, therefore, this condition is met when the phase of $z^{-N} H_{\mathrm{r}}(z)$ is a multiple of $2 \pi$ :

$$
\varphi\left\{z^{-N} H_{\mathrm{r}}(z)\right\}=\varphi\left\{e^{-j \vartheta_{k} N} H_{\mathrm{r}}\left(e^{j \vartheta_{k}}\right)\right\}=\quad-N \vartheta_{k}+\varphi\left\{H_{\mathrm{r}}\left(e^{j \vartheta_{k}}\right)\right\}=-k 2 \pi,
$$

which gives a digital angular frequency $\vartheta_{k}$ for each $k$. The analog partial frequencies become $f_{k}=\left[f_{\mathrm{s}} /(2 \pi)\right] \vartheta_{k}[35]$.

The decay time of mode $k$ having the frequency $f_{k}$ can be simply computed by knowing that mode $k$ is attenuated by $\left|H_{\mathrm{r}}\left(e^{j \vartheta_{k}}\right)\right|$ each time it passes the reflection filter. As one period of mode $k$ fits into the digital waveguide loop $k$ times, it is attenuated at a periodicity of $k / f_{k}$. This gives the following expression for the decay times:

$$
\tau_{k}=-k /\left(f_{k} \ln \left|H_{\mathrm{r}}\left(e^{j \vartheta_{k}}\right)\right|\right)
$$


where $\vartheta_{k}=\left(2 \pi f_{k}\right) / f_{\mathrm{s}} 35$.

Equations (8) and (9) show that the phase response of $H_{\mathrm{r}}(z)$ determines the frequencies of the string partials, while the magnitude of $H_{\mathrm{r}}(z)$ controls their decay time. This fact can be used to accurately tune the behavior of the partials by carefully designing a digital filter $H_{\mathrm{r}}(z)$ with the magnitude and phase responses obtained from the inverses of (8) and (9). The reflection filter is usually implemented as a low-order loss filter $H_{1}(z)$ (a first-order IIR lowpass is a common approximation) and an all-pass filter $H_{\text {ap }}(z)$ (orders between 5 and 20) in series.

The first step of this process is the analysis of real piano tones from which the partial frequencies and decay times are obtained, for example by STFT or heterodyne filtering [36]. Then the partial frequencies are used to decide on the number of delay elements $N$ and to design an allpass filter $H_{\text {ap }}(z)$ whose total delay leads to the synthesized partial frequencies (see (8) ) close to the original. Then the measured decay times are used to design a low-pass filter that attenuates the signal in every roundtrip in such a way that the synthesized decay times (given by (9p) are as desired. With this method it is possible to closely match the sonic properties of real piano tones, while the model still preserves the physical behavior of the string.

Modeling the effect of multiple strings belonging to the same key and the coupling of the two transverse polarizations would require the use of six digital waveguides whose vibration is also coupled at the termination. However, for efficiency, simplified models are used, e.g., running two or three waveguide models in parallel for the same note [37], a few resonators in parallel [36 or using modulated bandpass filters tuned to specific partials [38].

When the sustain pedal is pressed, the sounding notes excite all the unstruck strings as well: this can be taken into account by feeding signal from all string models to all the others. Care has to be taken not to create a positive feedback by such a connection. A common approach is that instead of feeding signal by trial and error, the physical equations are developed for all the strings connected to a passive termination [39,40]. When a physically passive system is discretized, the stability of the digital model is assured. Other, less physical approach is routing the string models in such a way that there is no feedback path. For example, this can be done by sending signals from the primary string models to the secondary ones, and not vice versa [38].

Coming from the efficiency of digital waveguides, this was the primary method for piano synthesis in the early times. The first waveguide-based piano model has been developed in 1987 [39], and other piano models with digital waveguides include [10,36 38, 40].

\subsection{Modal synthesis of string vibrations}

While digital waveguides are capable of the highly efficient modeling of linear string behavior, they are not very well suited to model nonlinear string vibration. On the other hand, the nonlinear longitudinal vibrations of the low and middle range of piano strings are very important for realistic bass piano sounds [41]. Because of this need, and by the help 
of increased computational resources, modal-based academic $17,35,42$ and commercia ${ }^{1}$ piano models have been developed around year 2005-2006.

Rather than the time-domain traveling wave solution, modal synthesis is based on the standing-wave solution of the wave equation and describes the motion of the string with a set of vibrational modes. The modal shapes of the ideal string with perfect boundary conditions are sinusoidal functions. The string displacement at any time instant can be expressed as the linear combination of these modal shapes:

$$
y(x, t)=\sum_{k=1}^{\infty} y_{k}(t) \sin \left(\frac{k \pi x}{L}\right) \quad x \in[0, L],
$$

where $y_{k}(t)$ is the instantaneous amplitude of mode $k$.

If $(10)$ is substituted into the wave equation (1a), then multiplied by the modal shape $\sin (k \pi x / L)$ and integrated over $x$ from 0 to $L$ (similarly to calculating the Fourier transform), all the derivatives with respect to space $x$ vanish and only time-derivatives remain. This results in an ordinary second-order differential equation governing the behavior of mode $k$

$$
\frac{d^{2} y_{k}}{d t^{2}}+a_{1, k} \frac{d y_{k}}{d t}+a_{0, k} y_{k}=b_{0, k} F_{y, k}(t)
$$

which is similar to the differential equation describing the vibration of a mass-springdamper system or an LRC circuit. The impulse response of such a system can be written analytically, and when damping is moderate, it is an exponentially decaying sinusoidal function

$$
y_{\delta, k}(t)=A_{k} e^{-\frac{t}{\tau_{k}}} \sin \left(2 \pi f_{k} t\right)
$$

The term $F_{y, k}(t)$ in (11) is the excitation force acting on mode $k$, and it is computed as the scalar product of the excitation force density and the modal shape. The exact values of the initial amplitude $A_{k}$, partial frequency $f_{k}$ and decay time $\tau_{k}$ can be computed by simple expressions from the physical parameters of the string (mass, stiffness, losses) [42].

The importance of splitting the partial differential equation of the string into simple second-order differential equations (11) lies in the fact that now each vibrational mode of the string can be modeled by a second-order resonator, which, in discrete time, becomes a second-order IIR filter that can be implemented very efficiently. In addition, the quality and the computational complexity can be easily scaled by the choice of the number of resonators. For the lowest tones this is in the order of a hundred, while for the highest ones, around five resonators are sufficient.

We note that the modal decomposition can be seen as a special discretization method with sinusoidal basis functions. Compared to the Finite Elements Method, an important computational benefit is that the stiffness matrix $A_{k, j}$ in (2) becomes diagonal because of the orthogonality of the basis functions. Thus, the equations describing the vibrations of

\footnotetext{
${ }^{1}$ Pianoteq software by Modartt, www.pianoteq.com
} 
the modes can be computed independently, as described above. On the other hand, since the basis functions are not localized in space, the reconstruction of the motion of the whole string would be computationally very expensive, but this is not needed in the context of sound synthesis where only the force at the termination of the string is required.

By the use of the impulse invariant transform, the discrete-time impulse response of a vibrational mode is obtained by simply sampling the continuous-time impulse response (12), yielding

$$
y_{\delta, k}[n]=y_{\delta, k}\left(t_{n}\right)=A_{k} e^{-\frac{t_{n}}{\tau_{k}}} \sin \left(2 \pi f_{k} t_{n}\right) / f_{\mathrm{s}},
$$

where $t_{n}=n T_{\mathrm{s}}, T_{\mathrm{s}}=1 / f_{\mathrm{s}}$ being the sampling interval. Equation $(13)$ differs from (12) by a scaling factor of $1 / f_{\mathrm{s}}$. This scaling is required because the discrete-time unit pulse has an area of $1 / f_{\mathrm{s}}$, while the continuous-time Dirac impulse has unity area.

Taking the $z$ transform of $y_{\delta, k}[n]$, after some algebra, gives

$$
\begin{aligned}
& H_{\mathrm{res}, k}(z)=\frac{b_{k} z^{-1}}{1+a_{1, k} z^{-1}+a_{2, k} z^{-2}} \\
& p_{k}=e^{j 2 \pi \frac{f_{k}}{f_{\mathrm{s}}}} e^{-\frac{1}{\tau_{k} f_{\mathrm{s}}}}, \quad b_{k}=\frac{A_{k}}{f_{\mathrm{s}}} \operatorname{Im}\left\{p_{k}\right\}, \\
& a_{1, k}=-2 \operatorname{Re}\left\{p_{k}\right\}, \quad a_{2, k}=\left|p_{k}\right|^{2} .
\end{aligned}
$$

That is, each mode is implemented by a two-pole filter and a delay in series, all connected in parallel, as displayed in Fig. 6. The input coefficients $w_{\mathrm{in}, k}$ are distributing the force input from the hammer $F_{\text {in }}$ to the different vibrational modes, while $w_{\text {out }, k}$ are the output weights for giving the force at the bridge $F_{\text {out }}$.

While the parameters of the vibrational modes, and thus the coefficients of the secondorder filters can be directly computed from the physical parameters of the string, it is also possible to set them directly, based on the analysis of recorded piano tones. Similarly to digital waveguides, this consists in estimating the frequencies and decay times of the partials and then using these in (14).

Compared to digital waveguides, one of the main benefit of the modal string model is the complete control of the behavior of partials that allows matching the sonic properties of a specific piano very accurately, a feature often desired by piano players. The other advantage is that the nonlinear longitudinal vibration responsible for the characteristic metallic sound of low piano strings can be very efficiently modeled by this technique as opposed to digital waveguides.

With second order accurate approximation, the longitudinal vibration of the string can be described by a similar equation as the transversal one (see (30), thus, it can also be modeled as a parallel set of second-order resonators (IIR filters). The longitudinal modes gain energy from the transverse motion of the string by a nonlinear coupling, and it turns out that a longitudinal mode with mode number $k$ is excited by the product of two transversal modes whose mode numbers $m$ and $n$ satisfy $k=m+n$ or $k=|m-n|$ [35, 42]. 


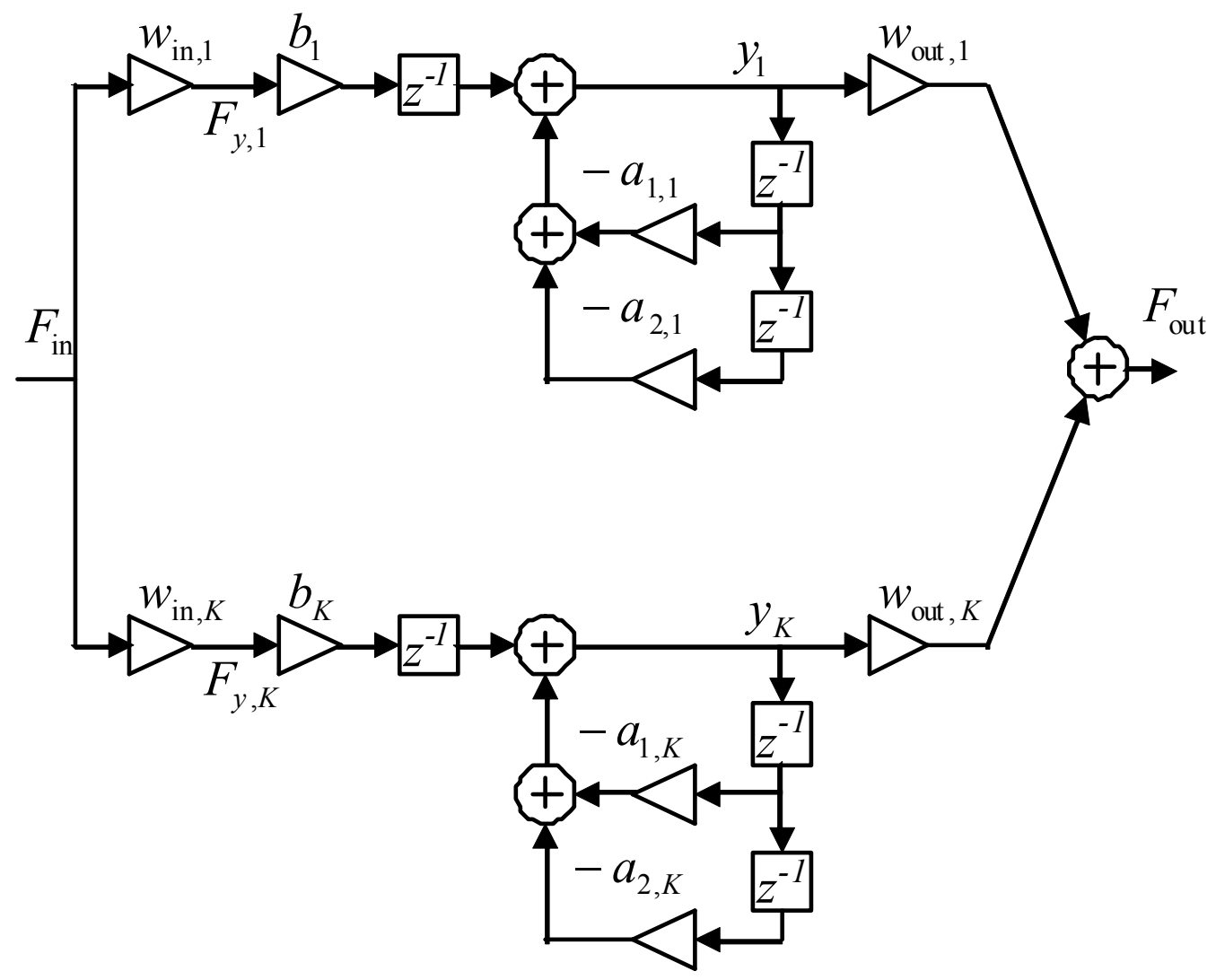

Figure 6: Modal based string model using second-order IIR filters in parallel. 
From a modeling point of view this means that the nonlinear longitudinal vibrations can be generated by cross-multiplying the output of the resonators of the primary (transverse) string model and leading this second-order signal to the resonators of the longitudinal string model.

The effect of the coupling of different strings belonging to the same note is again implemented by running more transversal string models in parallel, similarly to digital waveguides. However, since here the computational complexity scales linearly with the number of modes implemented, this secondary, less important string model may contain less resonators compared to the main one [42].

\subsection{Modeling the hammer}

For efficiency reasons the three-dimensional nature of the hammers is neglected in real-time synthesizers. One of the approaches is simply generating a signal that corresponds to the hammer shape either as a simple function (e.g., Hann window) or stored in a wavetable $[38,39]$. This has the benefit that the sonic properties of the resulting tone (loudness of the partials) can be directly controlled.

Another approach is to run a simplified physical model of the hammer $9,36,40,42$, with the benefit of a "more physical" behavior, required, e.g., for modeling the repeated strike of the same string.

The 0D hammer equations described in Sec. 3.2 can be easily discretized with respect to time. Equation (4a) is a static nonlinearity so it is implemented as is. Equation 4b can be converted to a discrete-time system by integrating (4b) with respect to time twice and then applying the impulse invariant transform. Thus, the discrete-time version of (4) is the following:

$$
\begin{aligned}
& F_{\mathrm{h}}[n]=F(\Delta y)=F\left(y_{\mathrm{h}}[n]-y_{\mathrm{s}}[n]\right), \\
& y_{\mathrm{h}}[n]=2 y_{\mathrm{h}}[n-1]-y_{\mathrm{h}}[n-2]-\frac{1}{m_{\mathrm{h}} f_{\mathrm{s}}^{2}} F_{\mathrm{h}}[n],
\end{aligned}
$$

where $f_{\mathrm{s}}$ is the sampling rate.

One interesting feature of $(15)$ is that there is a mutual dependence between $F_{\mathrm{h}}[n]$ and $y_{\mathrm{h}}[n]$. A simple remedy for this "delay-free loop" is inserting a unit delay between the equations, that is, using the past values of the variables, but this may lead to numerical instability. Accurate modeling requires the real-time solution of the two equations (15) for each time instant $n$ during the hammer is in contact with the string 40 .

\subsection{Modeling the soundboard and sound radiation}

The most expensive part of the piano from the modeling point of view is the piano soundboard since it involves a two-dimensional vibrating structure and computing the radiation in three dimensions. However, if we accept that we cannot change the physical parameters 
of the soundboard, a black-box model can be used to speed up the computations instead of complete model based on the material and geometric properties of the instrument.

The effect of the piano soundboard is twofold: first, it provides a termination to the strings together with the bridge, and thus influences the modal frequencies and decay times of the string partials and creates a coupling among them. This "termination" effect is usually included in the string model, since there it is easier to take into account, e.g., by modifying the mode decay parameters of the strings. The other effect is that the soundboard radiates the string vibrations which means amplification and frequency-dependent filtering. This latter "radiation" effect is the one that we consider in physics-based soundboard models. As a result we practically uncouple the string-soundboard system, and create a feedforward structure that is much more suitable for DSP implementation.

The computationally most efficient way of implementing the effect of the soundboard filtering is commuted synthesis [37, where the order of the model blocks (hammer-stringsoundboard) is commuted. By assuming the linearity of the model blocks, their order can be changed: now the impulse response of the soundboard excites the strings and the effect of the hammer is taken into account as a filtering operation. This method assumes linearity and time-invariance, therefore, some important effects, such as the restrike of the same string or nonlinear vibration of strings cannot be precisely modeled.

The impulse response of a piano soundboard is quite noiselike, similarly to the impulse response of a room, albeit with much shorter decay. Coming from this similarity, algorithms used to model room reverberation result in a very efficient way of modeling the filtering effect of the soundboard. Examples include coupled digital waveguides [39] and feedback delay networks [36]. The advantage is very low computational complexity, but the difficulty of the approach is setting the parameters of the reverberation algorithm in such a way that it results in the sound of a specific piano.

A very accurate way of modeling the effect of piano soundboard is to design a digital filter based on the measured vibration and radiation response of actual piano soundboards. This can be most simply done by an FIR filter, but more efficient approaches are available, including multi-rate FIR filtering [35], specialized IIR filter design [42], FFT-based convolution [42] and the combination of the two [43].

\section{Conclusion}

This paper has reviewed the main features of current piano models based on the physical description of the instrument. While these comprehensive models allow to understand the functioning of the instrument, the produced sounds are disappointing because many features are missing, but also because the some phenomena (as dissipation) are not yet accurately modeled.

Physics-based piano synthesis has three decades tradition in academic research, starting with a digital-waveguide based piano model in 1987 [39]. Digital waveguide has remained 


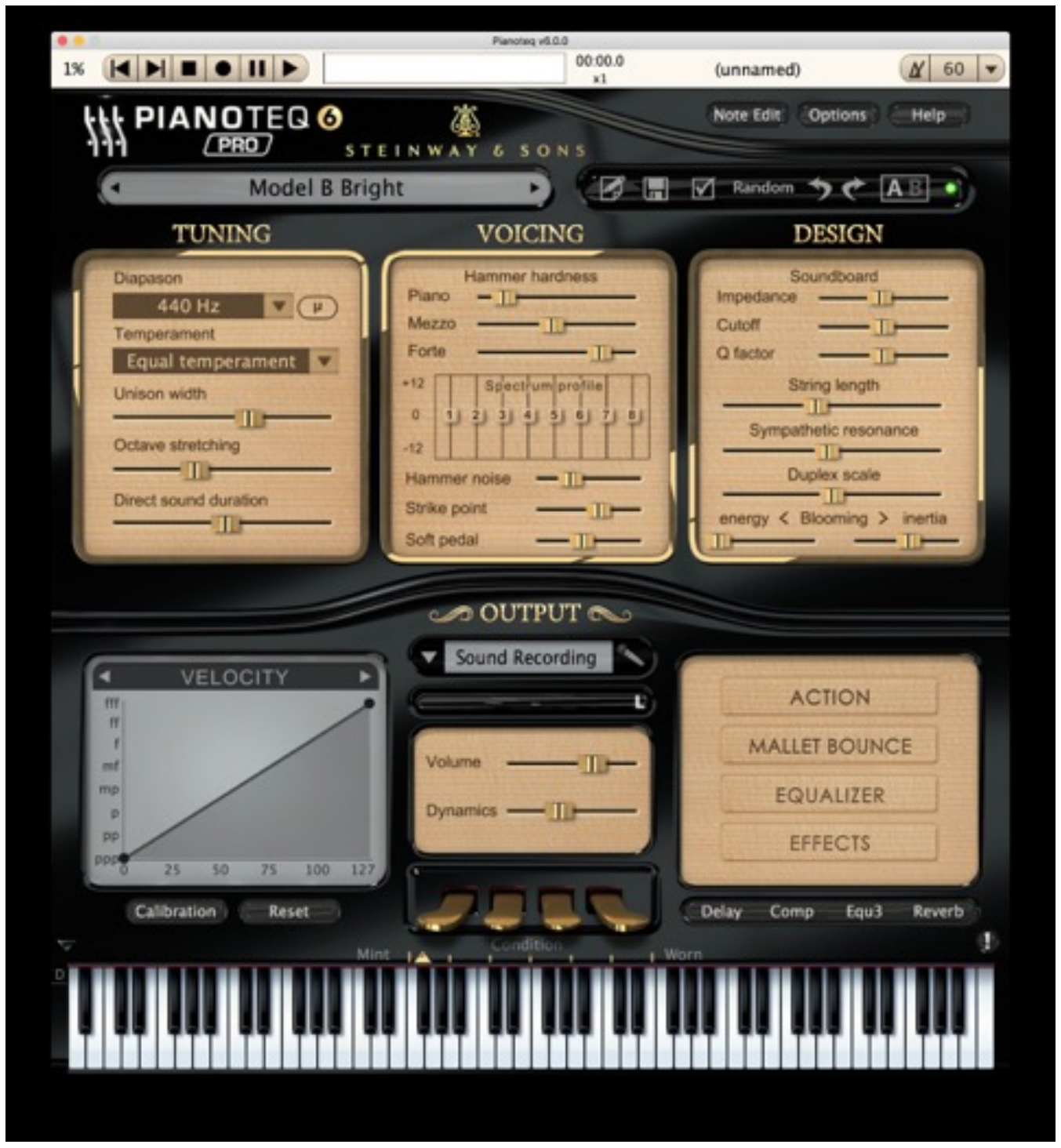

Figure 7: Pianoteq PRO 6 interface. The Pianoteq software computes the piano sound in real time using physical models. 


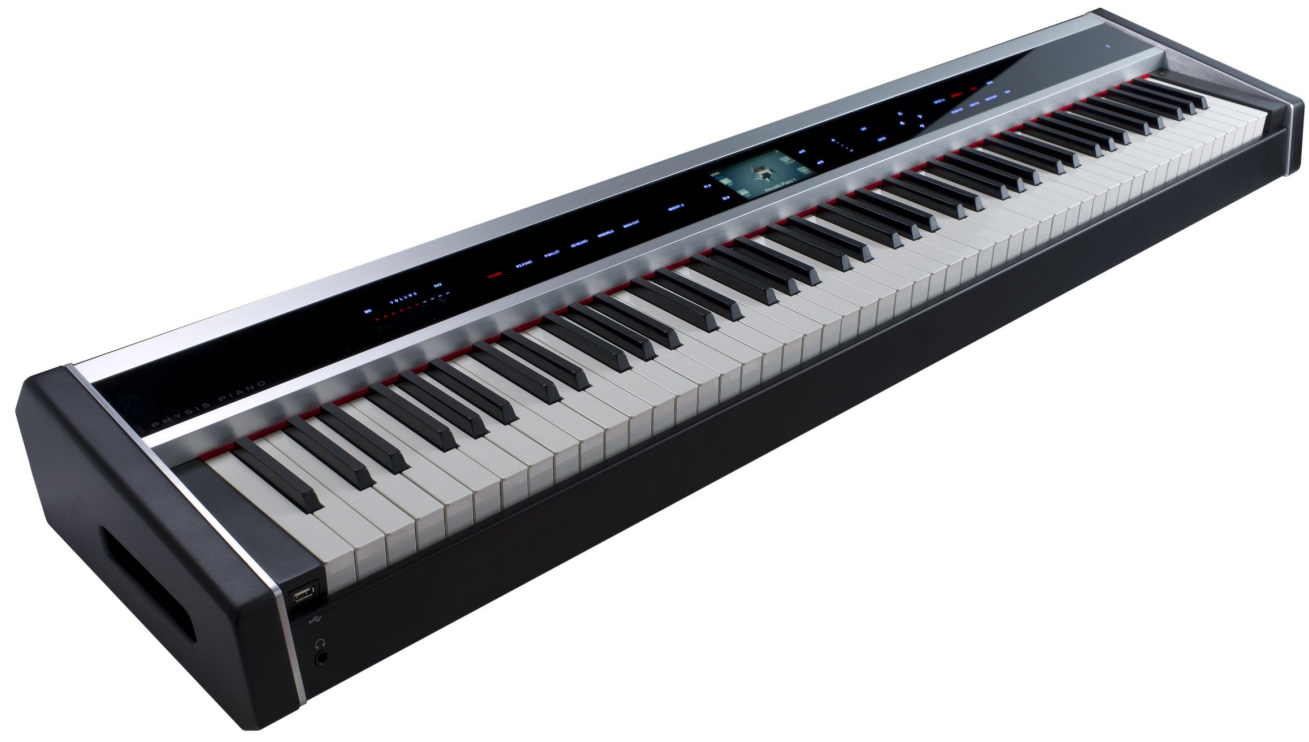

Figure 8: Physis Piano H1 from Viscount Corporation applying real-time modal synthesis.

the modeling paradigm for the next two decades. With the availability of more computational power and the need for modeling nonlinear string vibrations, a modal-based piano model appeared in year 2005 [17,35]. In parallel, a modal-based software piano, Pianoteq, was introduced by Modart $2^{2}$ in 2006 (see Fig. 7), and the first digital piano employing physical modeling was presented by Roland in 20093. Viscount has introduced the Physis piand ${ }^{4}$ in 2012, also using modal synthesis (see Fig. 8). By the availability of increased computational power it is expected that these existing models will continue to improve, and that other commercial products will be available that use physical-modeling for piano synthesis, as well as for other struck / plucked string instruments which have a similar physical functioning. Future research in piano modeling includes trying to better understand the string / soundboard coupling mechanism at the bridge, and how the pianist can influence the sound, but also to better model the shock of the key on the structure, or the effect of the crown on the soundboard vibrations. Regarding synthesis, future work will aim at further increasing the link between the model coefficients and the physical reality.

\footnotetext{
${ }^{2}$ Modartt, www.pianoteq.com

${ }^{3}$ Roland V-piano, www.roland.com/products/en/V-Piano

${ }^{4}$ Viscount corporation, www.physispiano.com
} 


\section{References}

[1] A. Askenfelt and E. V. Jansson, "From touch to vibrations. I: Timing in the grand piano action," J. Acoust. Soc. Am., vol. 88, no. 1, pp. 52-63, July 1990.

[2] — , "From touch to vibrations. III: String motion and spectra," J. Acoust. Soc. Am., vol. 93, no. 4, pp. 2181-2196, Apr. 1993.

[3] G. Weinreich, "Coupled piano strings," J. Acoust. Soc. Am., vol. 62, no. 6, pp. 1474-1484, Dec. 1977.

[4] H. A. Conklin, "Generation of partials due to nonlinear mixing in a stringed instrument," J. Acoust. Soc. Am., vol. 105, no. 1, pp. 536-545, Jan. 1999.

[5] M. Podlesak and A. R. Lee, "Dispersion of waves in piano strings," J. Acoust. Soc. Am., vol. 83, no. 1, pp. 305-317, Jan. 1988.

[6] H. A. Conklin, "Design and tone in the mechanoacoustic piano. Part I. Piano hammers and tonal effects," J. Acoust. Soc. Am., vol. 99, no. 6, pp. 3286-3296, June 1996.

[7] J. J. Tan, A. Chaigne, A. Acri et al., "Contribution of the vibration of various piano components in the resulting piano sound," in 22nd International Congress on Acoustics ICA 2016, 2016, pp. $1-10$.

[8] L. Hiller and P. Ruiz, "Synthesizing musical sounds by solving the wave equation for vibrating objects," Audio Eng. Soc., vol. 19, pp. 462-72, 542-51, 1971.

[9] A. Chaigne and A. Askenfelt, "Numerical simulations of piano strings. I. A physical model for a struck string using finite difference methods," J. Acoust. Soc. Am., vol. 95, no. 2, pp. 1112-1118, Feb. 1994.

[10] J. Bensa, S. Bilbao, R. Kronland-Martinet, and J. O. Smith, "The simulation of piano string vibration: From physical models to finite difference schemes and digital waveguides," J. Acoust. Soc. Am., vol. 114, no. 2, pp. 1095-1107, Aug. 2003.

[11] N. Giordano and M. Jiang, "Physical modeling of the piano," EURASIP J. on Applied Signal Process., vol. 2004, no. 7, pp. 926-933, June 2004.

[12] J. Chabassier, A. Chaigne, and P. Joly, "Modeling and simulation of a grand piano," The Journal of the Acoustical Society of America, vol. 134, no. 1, pp. 648-665, 2013.

[13] P. M. Morse and K. U. Ingard, Theoretical acoustics. Princeton university press, 1968.

[14] D. Rocchesso and F. Scalcon, "Bandwidth of perceived inharmonicity for physical modeling of dispersive strings," IEEE Trans. Speech Audio Process., vol. 7, no. 5, pp. 597-601, Sep. 1999.

[15] A. Quarteroni, R. Sacco, and F. Saleri, Méthodes Numériques, 2007.

[16] S. Bilbao, "Conservative numerical methods for nonlinear strings," J. Acoust. Soc. Am., vol. 118, no. 5, pp. 3316-3327, Nov. 2005.

[17] B. Bank and L. Sujbert, "Generation of longitudinal vibrations in piano strings: From physics to sound synthesis," J. Acoust. Soc. Am., vol. 117, no. 4, pp. 2268-2278, Apr. 2005. 
[18] S. Bilbao, "Energy-conserving finite difference schemes for tension-modulated strings," in Proc. IEEE Int. Conf. Acoust. Speech and Signal Process., Montreal, Canada, May 2004, pp. $\overline{285-}$ 288.

[19] J.-J. Tan, C. Touzé, and B. Cotté, "Double polarisation in nonlinear vibrating piano strings," in Vienna talk 2015 on music Acoustics, 2015.

[20] A. Izadbakhsh, J. McPhee, and S. Birkett, "Dynamic modeling and experimental testing of a piano action mechanism with a flexible hammer shank," Journal of computational and nonlinear dynamics, vol. 3, no. 3, p. 031004, 2008.

[21] A. Thorin, X. Boutillon, J. Lozada, and X. Merlhiot, "Non-smooth dynamics for an efficient simulation of the grand piano action," Meccanica, vol. 52, no. 11-12, pp. 2837-2854, 2017.

[22] X. Boutillon, "Model for piano hammers: Experimental determination and digital simulation," J. Acoust. Soc. Am., vol. 83, no. 2, pp. 746-754, Feb. 1988.

[23] A. Stulov, "Experimental and theoretical studies of piano hammer," in Proceedings of the Stockholm Music Acoustics Conference, vol. 485, 2003.

[24] J. Chabassier and M. Duruflé, "Energy based simulation of a Timoshenko beam in non-forced rotation. Application to the flexible piano hammer shank." Journal of Sound and Vibration, vol. 333, no. 26, pp. 7198-7215, 2014.

[25] A. Mamou-Mani, J. Frelat, and C. Besnainou, "Numerical simulation of a piano soundboard under downbearing," J Acoust Soc Am, vol. 123, p. 2401, 2008.

[26] E. Reissner, "The effect of transverse shear deformation on the bending of elastic plates," J. Appl. Mech., vol. 12, pp. 69-77, 1945.

[27] K. Ege, X. Boutillon, and M. Rébillat, "Vibroacoustics of the piano soundboard:(non) linearity and modal properties in the low-and mid-frequency ranges," Journal of Sound and Vibration, vol. 332 , no. 5, pp. 1288-1305, 2013.

[28] J. J. Tan, "Piano acoustics : string's double polarisation and piano source identification," Theses, Université Paris-Saclay, Nov. 2017.

[29] S. Bilbao, "Sound synthesis for nonlinear plates," in Proc. Conf. on Digital Audio Effects, Madrid, Spain, Sep. 2005, pp. 243-248.

[30] J. Chabassier, M. Duruflé, and P. Joly, "Time Domain Simulation of a Piano. Part 2 : Numerical Aspects," ESAIM: Mathematical Modelling and Numerical Analysis, vol. 50, no. 1, pp. 93-133, Jan. 2016.

[31] A. Chaigne, J. Chabassier, and M. Duruflé, "Energy analysis of structural changes in pianos," in Vienna Talk on Music Acoustics, Vienna, Austria, Sep. 2015.

[32] H. Järveläinen and T. Tolonen, "Perceptual tolerances for decay parameters in plucked string synthesis," J. Audio Eng. Soc., vol. 49, no. 11, pp. 1049-1059, July 2001.

[33] J. O. Smith, "Techniques for digital filter design and system identification with application to the violin," Ph.D. dissertation, Stanford University, California, USA, June 1983.

[34] T. I. Laakso, V. Välimäki, M. Karjalainen, and U. K. Laine, "Splitting the unit delay - tools for fractional delay filter design," IEEE Sign. Proc. Mag., vol. 13, no. 1, pp. 30-60, Jan. 1996. 
[35] B. Bank, "Physics-based sound synthesis of string instruments including geometric nonlinearities," Ph.D. dissertation, Budapest University of Technology and Economics, Hungary, Feb. 2006.

[36] — - "Physics-based sound synthesis of the piano," Master's thesis, Budapest University of Technology and Economics, Hungary, May 2000, published as Report 54 of HUT Laboratory of Acoustics and Audio Signal Processing.

[37] S. A. Van Duyne and J. O. Smith, "Developments for the commuted piano," in Proc. Int. Computer Music Conf., Banff, Canada, September 1995, pp. 319-326.

[38] J. Rauhala, H. M. Lehtonen, and V. Välimäki, "Toward next-generation digital keyboard instruments," IEEE Signal Process. Mag., vol. 24, no. 2, pp. 12-20, Mar. 2007.

[39] G. E. Garnett, "Modeling piano sound using digital waveguide filtering techniques," in Proc. Int. Computer Music Conf., Urbana, Illinois, USA, 1987, pp. 89-95.

[40] G. Borin, D. Rocchesso, and F. Scalcon, "A physical piano model for music performance," in Proc. Int. Computer Music Conf., Thessaloniki, Greece, Sep. 1997, pp. 350-353.

[41] B. Bank and H.-M. Lehtonen, "Perception of longitudinal components in piano string vibrations," J. Acoust. Soc. Am. Exp. Lett., vol. 128, no. 3, pp. EL117-EL128, Sep. 2010.

[42] B. Bank, S. Zambon, and F. Fontana, "A modal-based real-time piano synthesizer," IEEE Trans. Audio, Speech, and Lang. Process., vol. 18, no. 4, pp. 809-821, May 2010.

[43] S. Zambon, "Distributed piano soundboard modeling with common-pole parallel filters," in Proc. Stockholm Music Acoust. Conf., Stockholm, Sweden, Aug. 2013, pp. 641-647. 